\title{
REVIEW
}

\section{The role of TLRs in cervical cancer with HPV infection:}

\section{a review}

\author{
Xiao Yang ${ }^{1}$, Yanxiang Cheng ${ }^{1,2}$ and Chunsheng $\mathrm{Li}^{2}$
}

The main cause of cervical cancer is persistent infection with high-risk human papilloma virus (HR-HPV), but not all human papilloma virus (HPV) infections lead to cervical cancer. The key factors that determine the outcome of HPV infection remain poorly understood, and how the host immune system protects against HPV infection is unclear. Toll-like receptors (TLRs) are a group of pattern recognition receptors present in the cytoplasm and cell membrane, and can specifically recognize pathogen-associated molecular patterns. As the key molecules of innate and acquired immunity, TLRs not only play important roles in the immune defense against infectious diseases, but also are involved in the occurrence and development of a variety of malignant tumors. In cervical cancer caused by HR-HPV infection, TLRs have been found to regulate the local immune microenvironment. The role of TLRs in HR-HPV infection and HPV-induced cervical cancer and its relationship with HPV vaccine are reviewed in this article.

Signal Transduction and Targeted Therapy (2017) 2, e17055; doi:10.1038/sigtrans.2017.55; published online 3 November 2017

\section{INTRODUCTION}

Cervical cancer is one of the most common gynecological malignancies. The incidence of cervical cancer ranks second among women, and is a serious threat to women's health. ${ }^{1}$ Nearly all cervical cancer is caused by high-risk human papilloma virus (HR-HPV) infection, mainly HPV16 and HPV18. ${ }^{2}$ In most cases of human papilloma virus (HPV) infection, the HPV virus can be cleared by the immune system. However, persistent HPV infection develops in $10-15 \%$ of cases, and about $1 \%$ of HR-HPV infections eventually develops into cervical cancer. Persistent viral infection can be associated with the ability of HR-HPV to escape immune clearance, but the exact mechanism is unclear. HPV viruses can interfere with the expression of Toll-like receptors (TLRs) and regulate TLRs signaling pathways to induce persistent infection, which in turn leads to cervical lesions and eventually to cervical cancer (Figure 1).

\section{TLRS}

\section{TLR structure}

TLRs are highly conserved between humans and mice. To date, 11 human TLRs and 13 murine TLRs have been identified. ${ }^{3}$ TLRs are composed of an extracellular region, transmembrane region, and intracellular region, which are typical for transmembrane receptors. The extracellular region consists of 10 to 30 leucine-rich repeats, which can identify the pathogenic microorganism's pathogen-associated molecular patterns (PAMPs). The intracellular region contains approximately 200 amino acid residues, and has a high degree of homology with the cytoplasmic domain of interleukin-1 receptor (IL-1R). The cytoplasmic region plays an important role in downstream signaling. 4

\section{TLR distribution}

Based on subcellular localization, TLRs can be divided into two classes. One type localizes to the cell surface, and includes TLR1, 2, 4, 5 and 6, and mainly recognize the lipid composition of the surface of the pathogen. The other class of TLRs localize to the cytoplasm, and include TLR3, 7, 8 and 9, which mainly recognize the nucleic acid composition of the pathogen. ${ }^{5}$ TLRs are mainly expressed on innate immune cells, such as dendritic cells (DCs), mast cells, macrophages, neutrophils, endothelial cells and natural killer (NK) cells. ${ }^{6}$ In recent years, numerous studies have shown that TLRs are also expressed in tumor cells and cells in the tumor microenvironment of various cancers. In particular, TLR3, TLR4, TLR5 and TLR9 are frequently found in cervical cancer tissues. ${ }^{7-10}$ Furthermore, the activation of TLRs by different ligands plays important role in the development and progression of cervical cancer.

\section{TLR ligands}

TLR ligand specificities are determined by the extracellular amino acid composition of the receptors. TLRs can also recognize endogenous signals from damaged cells and their degradation products, such as heparin sulfate, and high mobility group box 1 protein. ${ }^{11}$ TLRs and their ligands are shown in Table 1.

\section{TLR signal transduction pathways}

At present, TLRs signal transduction pathways are divided into the human myeloid differentiation factor 88 (MyD88)-dependent pathway and MyD88-independent pathway. TLR1, TLR2, TLR5, TLR6, TLR7, TLR8 and TLR9 signals are mediated by MyD88-dependent pathway, and TLR3 signaling is by the MyD88-independent pathway, while TLR4 can activate and transduce signals through both pathways simultaneously. ${ }^{12}$

\footnotetext{
${ }^{1}$ Department of Obstetrics and Gynecology, Renmin Hospital of Wuhan University, Wuhan, China and ${ }^{2}$ Department of Obstetrics and Gynecology, University of Pennsylvania Perelman School of Medicine, Philadelphia, PA, USA.

Correspondence: Y Cheng or C Li (doctornancy@qq.com or chunsheng.li.phd@gmail.com)

Received 25 October 2016; revised 20 June 2017; accepted 17 July 2017
} 


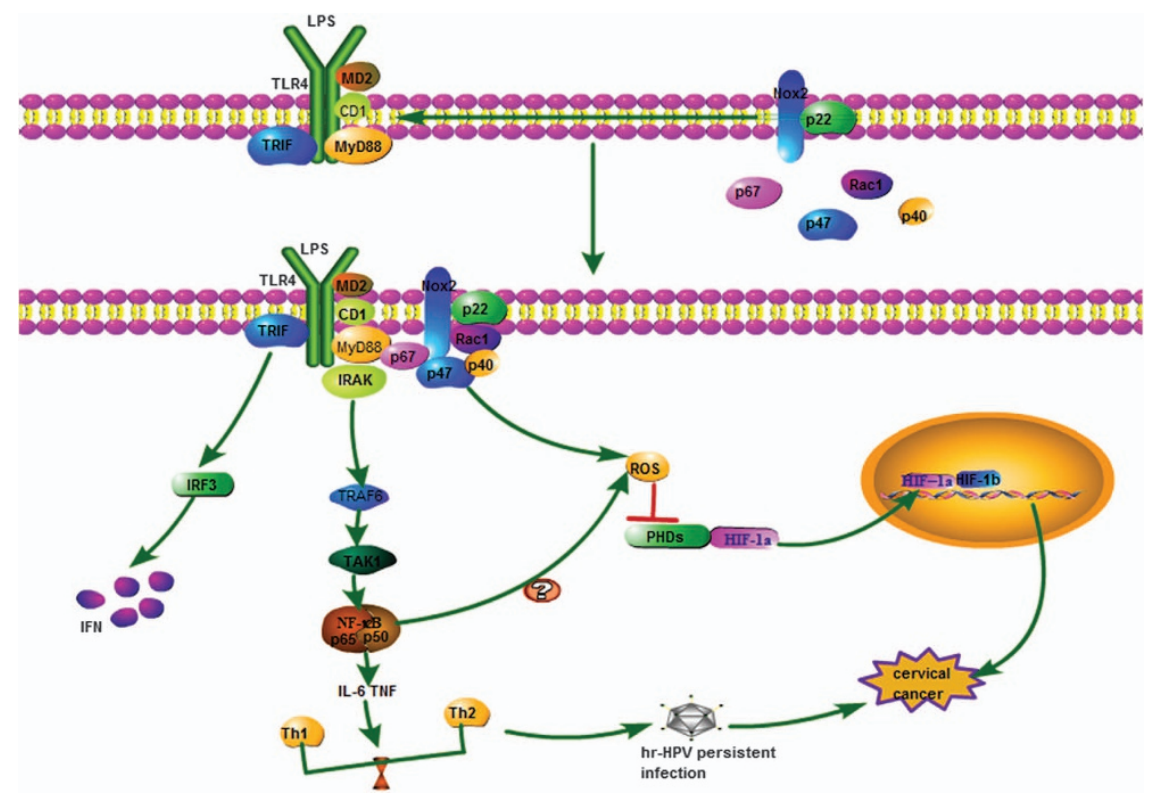

Figure 1. The possible mechanism of TLR4 being correlated with cervical cancer. The combination of TLR4 and its ligand (LPS) can trigger lipid rafts flowing which results in the change of lipid raft space conformation. This conformational change provides a condition for the aggregation of NADPH oxidase subunits on lipid rafts, which activates the redox reaction of lipid rafts to produce ROS and inhibits the degradation of HIF- $1 \alpha$, leading to the high expression of HIF-1 $\alpha$.

Table 1. TLRs and their ligands

\begin{tabular}{|c|c|}
\hline TLRs & Ligands \\
\hline TLR1/2 & Triacylated lipopeptides ${ }^{94}$ \\
\hline TLR2 & $\begin{array}{l}\text { Mycoplasma, non-lipopeptidic PAMPs from various } \\
\text { pathogens }{ }^{4}\end{array}$ \\
\hline TLR2/6 & Diacylated lipopeptides ${ }^{94}$ \\
\hline TLR3/7/8 & Viruses or bacteria mainly in the nucleic acid ${ }^{95}$ \\
\hline TLR4 & Lipopolysaccharide (LPS) of gram-negative bacteria ${ }^{94}$ \\
\hline TLR5 & Bacterial flagella ${ }^{96}$ \\
\hline TLR9 & Unmethylated DNA of bacteria or virus ${ }^{97}$ \\
\hline TLR10 & Remain unidentified \\
\hline TLR11 & T. gondii profilin and uropathogenic Escherichia coli ${ }^{98}$ \\
\hline
\end{tabular}

Abbreviations: PAMP, pathogen-associated molecular pattern; TLR, Toll-like receptors.

TLR3 and TLR4 can activate NF-KB in cells without MyD88. ${ }^{13,14}$ Also, TLR3 and TLR4 have been shown to activate IRF-3 and induce IFN-stimulated regulatory elements through a MyD88-independent signaling pathway. ${ }^{15,16}$ After binding to ligands, TLRs activate signal transduction cascades either via myeloid differentiation factor 88 (MyD88) pathway or the TIR joint protein molecules (TRIF) pathway, causing the activation of nuclear factor kappa B (NF-KB), mitogen activated protein kinase (MAPK), and other signaling pathways in a variety of immune cells. Eventually, activation of these pathways can induce the expression of a series of functional molecules, such as cytokines, trend factor and inflammatory factor, and initiate immune responses to enable natural defenses. There are at least three mechanisms in which TLRregulated inflammatory response can promote carcinogenesis: via activating the anti-apoptotic effect of NF-KB, a transcription factor important in inflammatory conditions, via causing oxidative damage to DNA, and via inducing the tissue repair response $^{17}$ (Figure 2 ).

\section{RELATIONSHIP BETWEEN TLRS AND CERVICAL TUMOR IMMUNITY}

TLRs and immunity

After recognizing microbial pathogens and other ligands, TLRs can induce DCs and macrophages to produce type 1 interferon and inflammatory cytokines. Activated TLRs can also induce DC maturation and enhance the antigen-presenting ability of DCs. TLRs can also regulate the proliferation and differentiation of Th1 cells and Th2 cells, which in turn establish resistance to external pathogen invasion. While most inflammatory mediators induced by TLRs can help to remove pathogens, excessive inflammatory mediators may lead to the occurrence of pathological inflammation, such as autoimmune disease, rheumatoid arthritis, inflammatory bowel disease and system lupus erythematosus. ${ }^{18}$ TLRs and T helper cell responses as shown in Figure 3. Macrophages and DCs can be inhibited via TNFAIP3, also termed tumor necrosis factor inducible protein A20, PTPN22, also termed LYP, or the BCAP/PI3K pathway. These three mechanisms can negatively regulate TLR-mediated immune response and influence the pathogenesis and treatment of autoimmune diseases. $^{19-21}$ LYP in mononuclear cells can downregulate the activity of TLRs, and LYP inhibits TLR responses through the inhibition of the TREM2/DAP12 pathway. $^{22}$ In aggregate, TLR activation can be a double-edged sword to the well-being of an organism. To maximize the benefit and harness the damage of TLR activation, greater efforts are required to understand the role of TLRs in immunity.

\section{TLRs and tumors}

TLRs are important components of the human immune system. They play important roles in combating viruses, bacteria, and other infections, and function in anti-tumor immune activity. Some recent studies have found that TLRs could facilitate the initiation, progression, and metastasis of tumors. In recent years, more and more studies have confirmed that TLRs expressed in tumor tissues with different subtypes of TLRs, such as gastric cancer, ${ }^{23,24}$ colorectal cancer, ${ }^{25,26}$ ovarian cancer, ${ }^{27}$ lung cancer, ${ }^{28}$ 


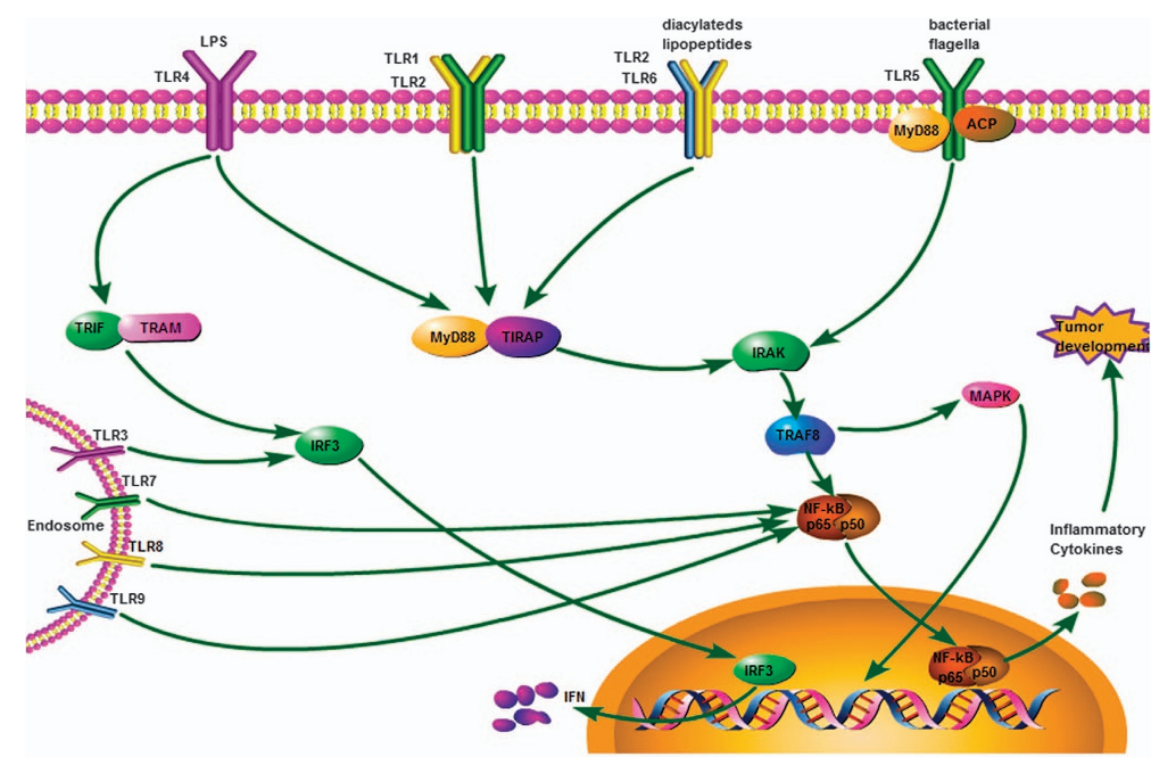

Figure 2. Toll-like receptor signaling. The MyD88-dependent pathway results in nuclear translocation of NF-kB and induction of pro-inflammatory cytokines. TLR3 and TLR4 activate IRF-3 and induce IFN-stimulated regulatory elements through a MyD88-independent signaling pathway.

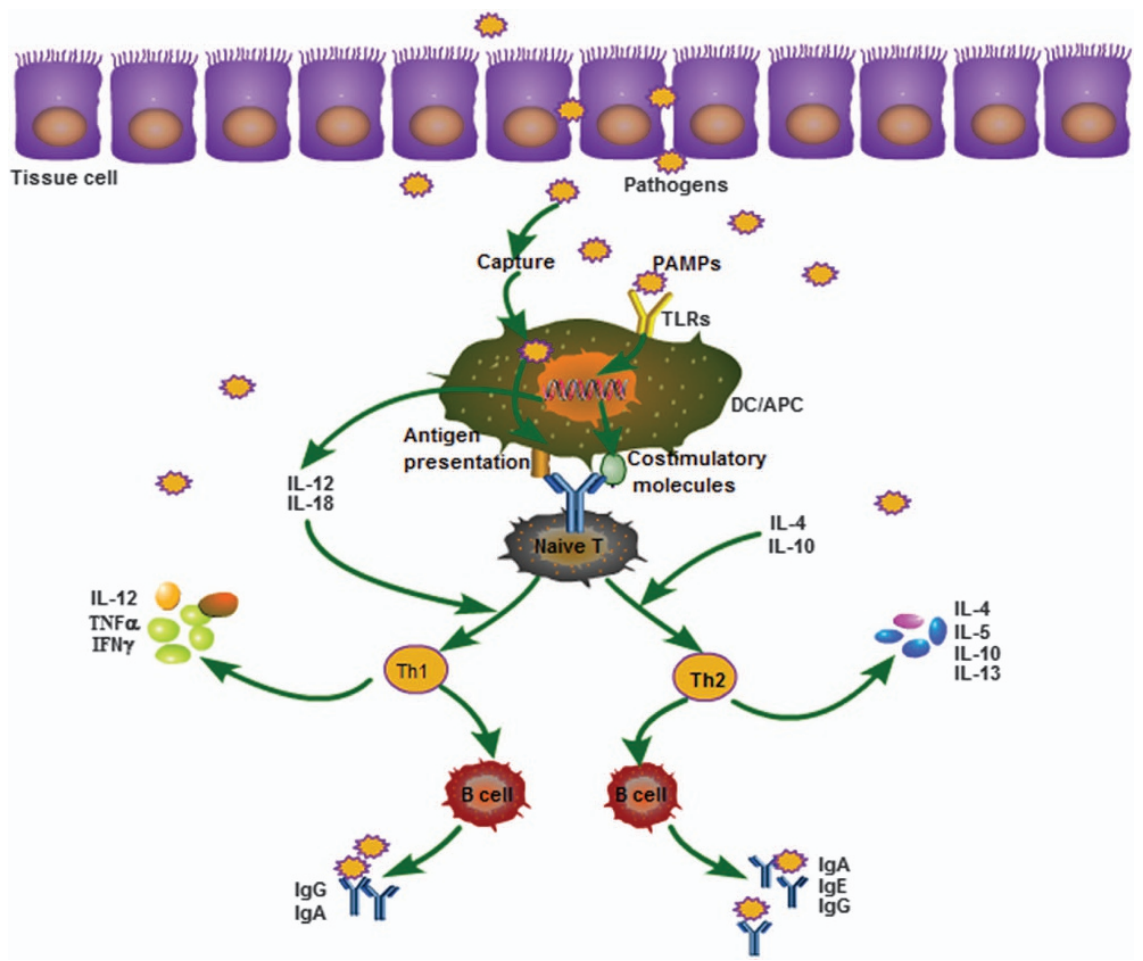

Figure 3. TLRs and T helper cell responses. PAMPs from invading pathogens bind with TLRs expressed in DCs, and DCs become activated and mature to active naive T cells. Naive T cells are primed toward specific T helper profiles: Th1, Th2, which can produce cytokines and in turn establish resistance to external pathogen invasion.

prostate cancer, $^{29}$ breast cancer, ${ }^{30,31}$ liver cancer $^{32,33}$ and pancreatic cancer, ${ }^{34}$ which were actived by different ligands playing an important role in the occurrence and development of tumor. The expression of TLRs in different tumor tissues is shown in Table 2. Furthermore, tumor development often is associated with abnormal expression of TLRs and chronic inflammation, yet the exact mechanism is not clear. de Matos et al. ${ }^{35}$ found that the expression levels of TLR2 and TLR4 were higher in cervical cancer and premalignant lesions compared to normal controls. Studies also found that TLRs, especially TLR4, TLR5 and TLR9, are closely related to HPV infection and cervical cancer. Activation of TLR4 can promote the growth of ovarian cancer cells. ${ }^{36}$ The endogenous TLR4 activator S100A8/S100A9 could promote growth and metastasis of tumors. ${ }^{37}$ HPV16 infection will activate the innate immune system and initiate an inflammatory reaction in cervical epithelium, which not only identifies and clears the HPV virus but can also promote cervical intraepithelial neoplasia, ultimately leading to cancer. Hasan et al. ${ }^{38}$ indicated that the HPV16 E6 protein inhibited TLR9 transcriptional pathways, affecting the immune systems' ability to recognize pathogens, thereby enabling 
virus to escape from immune surveillance. However, this phenomenon was not observed in the transfection of HPV18 E6 into cells, ${ }^{38}$ suggesting that different types of HR-HPV mediate different regulatory mechanisms on TLR expression in tumor development. Studies show that TLR3 mRNA was increased in cervical atypical squamous epithelium. TLR1 mRNA was decreased in cervical squamous cell carcinoma, yet the expression levels of TLR1, 2, 5, 6 and 9 were increased in parallel with the increase in the level of cervical lesions. ${ }^{39}$ This result suggests that there are specific temporal and spatial regulations of TLR expression in the different stages of lesion tissue and of cervical cancer development. However, the mechanism of how TLRs are involved in regulating the outcome of $\mathrm{HR}-\mathrm{HPV}$ infection has not been clarified. The relationship between TLRs and HPV can be accurately studied by subdividing the type of HPV, the type of TLRs, the grade of cervical lesions, and the composition of the tissues.

\section{TLR4 and cervical cancer}

In recent years, multiple studies showed that hypoxia is often present in solid tumors, and hypoxia plays a very important role in tumor occurrence, progression, malignant invasion, distant metastasis, and tumor resistance to radiotherapy and chemotherapy. Our previous studies showed that the expression of TLR4 in cervical cancer is related to the occurrence, development, and growth of cervical cancer cells. Uncontrolled growth of cervical

\begin{tabular}{|lc}
\hline Table 2. The expression of TLRs in different tumor tissues \\
\hline Tumor tissues & \multicolumn{1}{c}{ Expression of TLRs } \\
\hline gastric cancer & TLR2, TLR4, TLR5, TLR9 \\
colorectal cancer & TLR2, TLR3, TLR4, TLR5, TLR9 \\
ovarian cancer & TLR2, TLR3, TLR4, TLR5 \\
lung cancer & TLR2, TLR3, TLR4, TLR9 \\
prostate cancer & TLR4, TLR9 \\
breast cancer & TLR2, TLR3, TLR4, TLR9 \\
liver cancer & TLR2, TLR3, TLR4, TLR6, TLR9 \\
pancreatic cancer & TLR2, TLR4, TLR9 \\
\hline
\end{tabular}

Abbreviation: TLR, Toll-like receptors. cancer cells is closely related to the excessive activation of an important transcription factor, hypoxia-inducible factor-1a (HIF-1a). The cervical TLR4 signaling pathway is positively correlated with high expression of HIF- $1 a^{40}$ There is a hypoxic microenvironment in tumor tissues, and this hypoxic microenvironment can induce high expression of HIF-1a. However, the expression of HIF-1a and major histocompatibility complex class I -related chain $A / B(M I C A / B)$ are negatively correlated in cancer tissues. MICA/B are rarely expressed by normal cells, however, they are expressed in a variety of malignant diseases. Thus, these observations indicate that hypoxia is associated with immune escape of tumors. ${ }^{41}$ Tannahill et al. ${ }^{42}$ found that the activation of TLR4 via LPS strongly increases the levels of the TCA cycle intermediate succinate which is known to stabilize HIF-1a. Perhaps in the context of cancer an endogenous danger signal can use a similar mechanism of action to stabilize HIF-1a. ${ }^{42}$ This mechanism of action must be explored to provide a theoretical basis for finding targets for anticancer therapy.

TLR4 and the iNOS signaling pathway in cervical cancer. TLR4 activates signal transduction by the MyD88-dependent and MyD88-independent pathways, which in turn modulate inducible nitric oxygen synthase (iNOS) through tumor necrosis factor receptor-associated factor 6 (TRAF6), MAPK, NF-KB and other key genes. ${ }^{43}$ Rahkola et al. ${ }^{44}$ found that patients with HR-HPV infection have higher nitric oxide (NO) in the cervical canal than do HPVnegative patients, indicating that the release of $\mathrm{NO}$ is closely related to HPV infection. The expression of iNOS in prostate cancer, head and neck squamous cell carcinoma, gynecological tumors, ${ }^{45}$ breast cancer, and lung cancer tissues was different from that of normal tissue. In prostate cancer, the expression of iNOS was related to tumor cell proliferation. ${ }^{46}$ In breast cancer, iNOS and NO can stimulate the expression of ERBB2 and bFGF and promote the proliferation of breast cancer cells. ${ }^{47}$ In cervical cancer, iNOS expression was positively correlated with tumor clinical stage, malignant degree, distant metastasis, and tumor microvessel density. When iNOS activity was inhibited, the growth of tumor cells was also inhibited. ${ }^{48}$ Xiao et al. ${ }^{49}$ detected the mRNA and protein expression of TLR/iNOS pathway transduction molecules including TLR4, NF-KBp65 and iNOS in different cervical

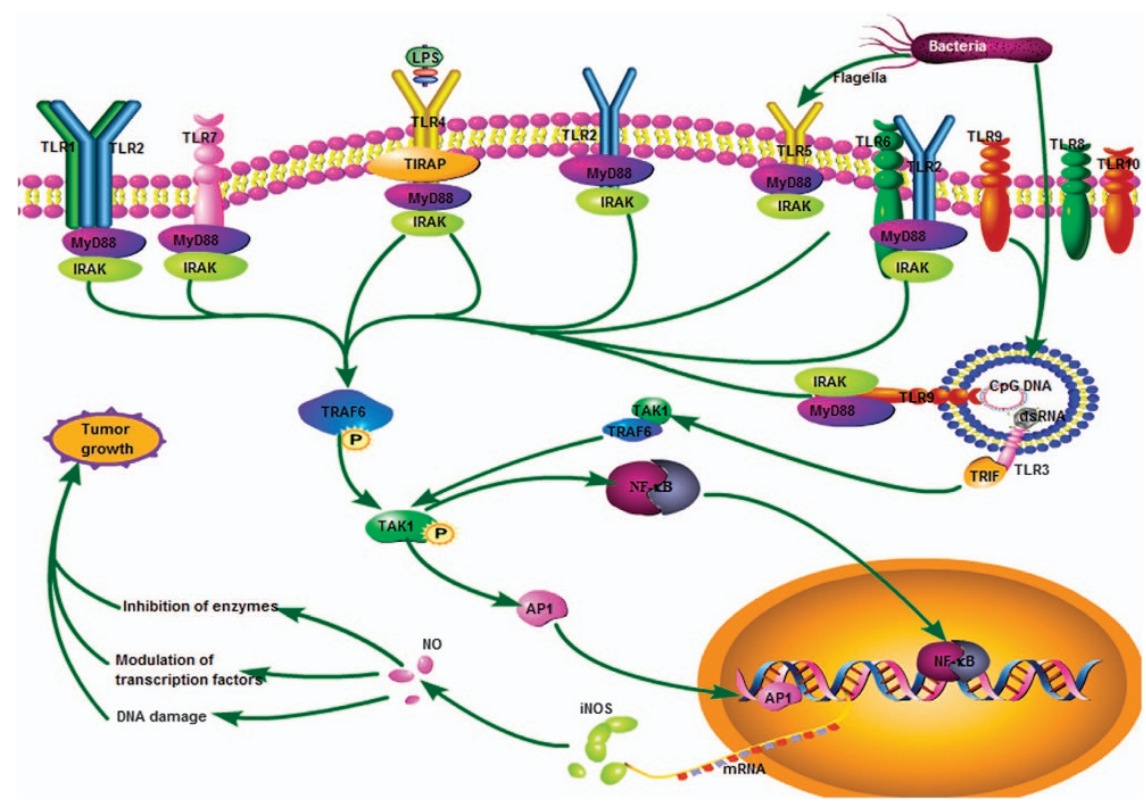

Figure 4. Synthesis of NO via TLR signaling. The TLRs are expressed on the cell surface or inside cells. TLRs use MyD-88 for downstream signaling, but TLR3 uses TRIF protein as an adaptor molecule. In downstream signaling, the activation of NF-кB or AP-1 can upregulate gene transcription for iNOS in the nucleus, and generate highly reactive NO, which can induce some effects and promote the proliferation of tumor. 
cell lines, and they found TLR4/iNOS pathway is highly expressed in cervical cancer with hrHPV infection which may be resulted in cervical tumorigenesis. Synthesis of NO via TLR signaling as shown in Figure 4. However, the mechanisms of how the cervical local microenvironment regulate the expression of iNOS in cervical lesions of different stages, thereby affecting HR-HPV infection and disease prognosis, and how HR-HPV escapes the cytotoxicity of high concentrations of NO upon iNOS induction, remain unclear. The answers to the above questions will help us to further understand the process of immune clearance of HR-HPV and the HR-HPV carcinogenic mechanism.

TLR4 and the NF-KB signaling pathway and cervical cancer. In recent years, NF-KB has been the subject of intense research interest and has become an attractive drug target. The regulation of NF-KB activity in cells could treat certain inflammatory diseases and control tumor progression. ${ }^{50} \mathrm{NF}-\mathrm{KB}$ is downstream of TLR4 signaling, controlling expression of various cytokines and inflammatory factors. The TLR/MYD88/NF-KB signaling pathway plays an important role in inflammation, tumor development and invasion. ${ }^{51}$ Our previous studies showed that TLR4 expression was substantially elevated in cervical carcinoma. ${ }^{40}$ Moreover, high TLR4 expression further activated NF-KB regulated inflammatory signaling pathways, including the HIF-1a signal pathway that promoted the production of the immunosuppressive cytokines IL-6, TGF- $\beta 1$, and others, which promoted the growth of Hela cells and enhanced their resistance to apoptosis. ${ }^{50}$ Xue-feng et al. showed that BPA induced migration of cervical cancer cells through the IKK- $\beta / N F-K B$ pathway, presumably by upregulating expression of metalloproteinase-9 (MMP-9) and fibronectin (FN) in Hela cells and Siha cells. Inhibition of NF-KB could eliminate the BPA-mediated upregulation of MMP-9 and FN. However, there were no significant changes of PKA, ERK1/2, EGFR or PI3K/AKT. ${ }^{52}$ Thus further investigation of TLR4 and the NF-KB pathway and their mechanism of action will help provide new ideas in developing therapeutic strategies to treat cervical cancer.

\section{TLR9}

TLR9 ligands include unmethylated deoxycytidyl-deoxyguanosin dinucleotide $(\mathrm{CpG})$ motifs in bacterial and viral DNA and oligonucleotide CpG motifs. The TLR9 signaling pathway is a
MyD88-dependent signal transduction pathway. TLR9 can initiate signal transduction by directly combining with CpG oligodeoxynucleotides (ODN) and increasing MyD88. This activation leads to the expression of endothelial adhesion molecules, inflammatory cytokines, interferon and co-stimulatory molecules, and plays an important role in the process of immunity, cell apoptosis, and antiinfection. ${ }^{53}$

Lee et $a l^{54}$ found that compared with the normal cervical epithelium, the expression of TLR9 in cervical cancer was significantly increased. Cannella et al. $^{55}$ found that TLR9 levels were much higher in patients persistently positive to the same HPV genotype, compared with women who cleared HPV infection and with those re-infected with a different genotype. This finding indicates that the increased TLR9 levels without HPV clearance in persistently infected women could drive inflammation, thereby contributing to cervical cancer risk. Jiang et al. showed that CpG ODN is a specific agonist of TLR9, activating the TLR9 pathway by upregulating transcription of TLR9 to play immunoregulatory effects. ${ }^{56} \mathrm{Di}$ et al. ${ }^{57}$ found that using the specific ligand of TLR9, CpG ODN, to stimulate the A549 lung cancer cell line can produce resistance to apoptosis induced by TNF-a. Therefore, TLR9 is beneficial to the survival of tumor cells. However, the mechanism of the role of TLR9 in the development of cancer remains to be elucidated.

\section{IMMUNE ESCAPE MECHANISM OF HPV IN CERVICAL LESIONS}

\section{Structure and function of HPV}

Humans are the only host of HPV, which mainly infects the skin and mucosal epithelial cells, with viruses being divided into skin types and mucosal types. Bosch and Manos et al. detected cervical cancer tissues from 22 countries by PCR, they found that HPVDNA were expressed in $99.7 \%$ of the tumors with no significant difference between countries.Research also found, HPV16 type 18 infection rate was the highest in all types, HPV16 accounted for $50 \%$, HPV 18 accounted for $14 \%$, HPV45 accounted for $8 \%$, HPV31 accounted for $5 \%$, other types of HPV accounted for $23 \%$. The type of HPV was associated with the pathological type of cervical cancer. HPV16 occupied the main position in cervical squamous cell carcinoma ( $51 \%$ squamous cell carcinoma specimens), and HPV18 occupies the main position in the adenocarcinoma of the

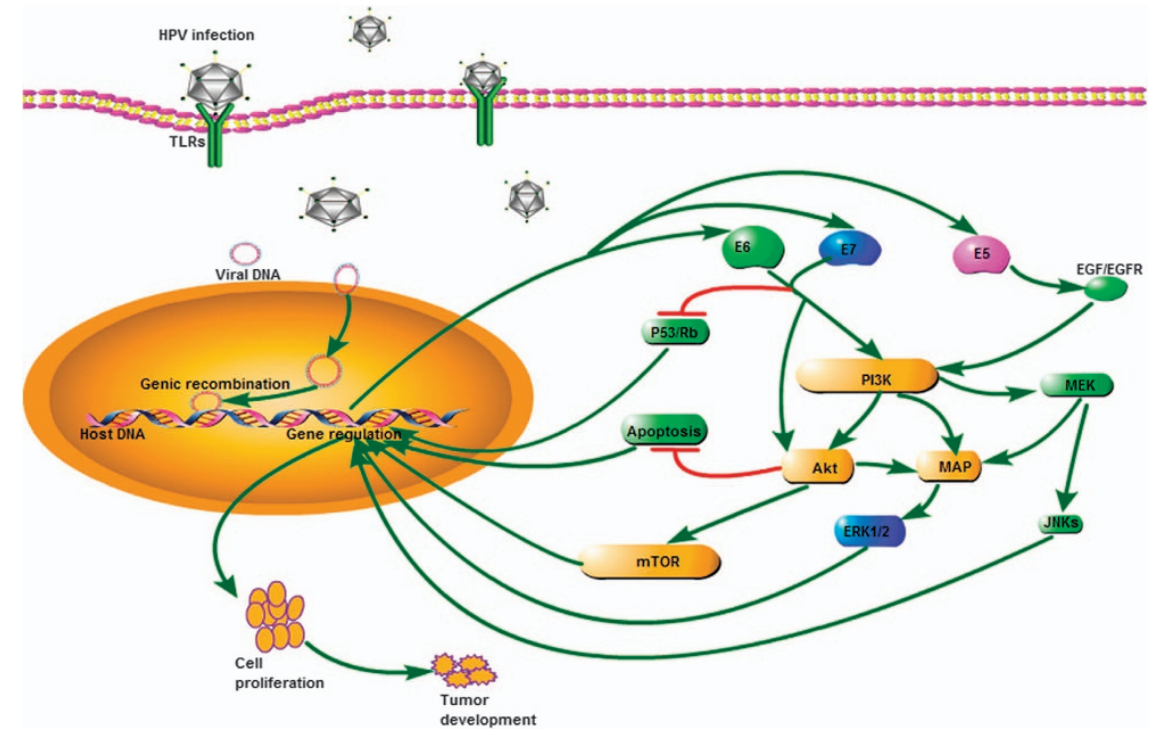

Figure 5. HPVs infect host epithelial cells through TLRs. The HPVs DNA viral replication is synchronous with host cellular DNA replication. The cells express E6/E7/E5 oncoproteins to damage the infected cells by inhibiting the expression of tumor suppressors p53 and Rb and decreasing apoptosis. E6/E7/E5 oncoproteins can also activate the PI3K/Akt/mTOR signaling pathway. All these processes enhance cell proliferation and promote tumor growth. 
uterine cervix $(56 \%$ specimens of glandular epithelial cell carcinoma) and cervical glandular squamous cell carcinoma (39\% specimens of adenosquamous cell carcinoma). ${ }^{58,59}$ Multiple studies have confirmed that HPV is detected in $98.5 \%$ of invasive cervical cancer, suggesting that HPV infection is closely linked to cervical cancer. ${ }^{60}$

HPV is a circular double-stranded DNA virus with a nonenveloped icosahedral structure. A total of 100 subtypes of HPV, such as HPV16, HPV18, HPV32, HPV52, HPV58 and so on, have been identified, and 38 can infect the genital tract. Depending on the oncogenic ability, HPV can be divided into low-risk and highrisk types. Low-risk HPV mainly causes benign lesions, and HRHPV, such as HPV16 and HPV18, can lead to malignant tumors. ${ }^{61}$ With persistent infection of HR-HPV in cervical epithelial cells, viral genes can be integrated into the host genome, resulting in squamous intraepithelial lesions through a cascading process. The HPV E2 gene encodes the early proteins which suppress the expression of E6 and E7. E6 and E7 proteins can inhibit the expression of tumor suppressor genes p53 and $\mathrm{Rb}$, resulting in malignant transformation of the host cell and eventually the occurrence of cancer. ${ }^{62}$ HPVs infect host epithelial cells through TLRs as shown in Figure 5.

The relationship between HPV infection and immune escape Cervical cancer is characterized by persistent infection with HPV. In some cases, however, the HPV virus can escape from immune surveillance, the mechanism of which remained unclear until recently. The immune system is crucial for virus elimination. Macrophage, NK cells and T lymphocytes all play important roles as the first-line defenders in eliminating HPV infection. The second line of defense is cytotoxic T lymphocytes (CTLs) inducing an adaptive immune response against HPV16 E6 and E7 to eliminate HPV infection. ${ }^{45}$ However, HPV can escape immune attack through a variety of mechanisms. First, HPV does not kill host cells during viral replication, and therefore does not present viral antigen nor induce inflammation. HPV16 E6 and E7 proteins can downregulate the expression of type 1 interferons (IFN-1) in host cells. IFN regulatory factor-1 (IRF-1) can induce the production of antigen presentation transporter (TAP-1), IFN- $\beta$ and monocyte chemotaxis protein-1 (MCP-1), which is very important for cellular immunity and plays a role in the prevention of inflammation in infected sites. The lack of co-stimulatory signals by inflammatory cytokines, including IFNs, during antigen recognition may induce immune tolerance rather than the appropriate responses. Moreover, HPV16 E5 protein downregulates the expression of HLA-class 1, and reduces recognition by $\mathrm{CD} 8 \mathrm{~T}$ cells. ${ }^{63}$ The lack of cytokine production in HPV-infected sites prevents the activation and maturation of DCs, thus inhibiting the activation of a positive CTL response. ${ }^{64}$

At the same time, it was showed that HPV E5 and E7 proteins inhibited the expression of major histocompatibility complex class I proteins on the cell surface. HPV-infected cells are resistant to lysis by NK cells, but are sensitive to cytokine-activated NK cells. Activated macrophages play an important role in the elimination of HPV-infected cells and control the development of tissue malignancy. ${ }^{65}$ Therefore, innate immunity plays a key role in the prevention and elimination of HPV infection, but immune escape and inactivation of innate immunity still occur, which may eventually lead to persistent HPV infection, and further lead to the occurrence of cervical cancer.

The relationship between HPVE6 protein and cervical cancer HPV infection can lead to a series of biochemical changes, such as regulation of cell cycle, virus copy, apoptosis, cell proliferation and cell malignant phenotype. All of these can be regulated via the E6 and E7 proteins. $^{62}$ The expression of the HPV16 E6 protein is closely related to the occurrence of tumors. E6 protein plays a role in HPV infection through a variety of mechanisms. It binds to P53 and accelerates the degradation and inactivation of that tumor suppressor, thereby inhibiting cell apoptosis and promoting the occurrence and development of tumors. ${ }^{66}$ It regulates the activity of telomerase, thereby inhibiting the normal process of cell apoptosis and promoting cell immortalization. ${ }^{63}$ It can also cooperate with the death domain-associated protein (DAXX) to inhibit apoptosis. ${ }^{67}$ The HPV16 E6 protein also interacts with IFN-3 to downregulate the expression of IFN- $\alpha$ and IFN- $\beta$; it inhibits the antiviral activity of IFNs and downregulates TNF- $\alpha$ and IL-1 $\beta$ secreted by macrophages following activation by LPS, both of which help tumor cells escape from the innate immune response of the host. ${ }^{68}$ HPV16 E6 protein is closely related to the pathogenesis of cervical cancer, so it is very important to understand the biological function of E6 and to study its effect on cervical cancer and its mechanism.

\section{CORRELATION BETWEEN HPV ESCAPE FROM IMMUNE CLEARANCE AND TLRS}

Chronic inflammation is closely related to tumor development and progression. ${ }^{69}$ However, the tumorigenic mechanism of chronic inflammation is unclear. Xiao et al. initially explored the expression of the TLR/NO pathway in HR-HPV-positive cervical cancer tissues. They found that the key genes of TLR4/NO pathway, such as TLR4, NF-KBp65, and iNOS, are highly expressed in HR-HPV-positive cervical cancer tissues and cells, ${ }^{49}$ strongly suggesting that the TLR4/NO signal pathway may be associated with the HR-HPV infection. This pathway may mediate alterations of the local immune microenvironment in cervix, facilitating immune escape or immune tolerance of tumors, eventually leading to the occurrence, development, and metastasis of cervical cancer. Specific mechanisms of the TLR4/NO pathway and HR-HPV involved in cervical cancer need further exploration.

Intriguingly, some studies revealed that the NO content with HR-HPV infection was significantly higher than that of low-risk HPV infection and no HPV infection, thus the NO content of the cervical canal was closely related to the HR-HPV infection status. ${ }^{70}$ Daud et al. found that cervical epithelial cells with low expression of TLR9 were more susceptible to HPV infection, and cervical epithelial cells with high TLR9 expression rarely have any HPV infection, suggesting that the elimination of HPV infection may involve the increased expression of TLR9. ${ }^{71}$ Hasan et al. showed that HPVE6 protein can downregulate the expression of the TLR9 gene and inhibit the expression of TLR9 signaling pathway related molecules, thus affecting identification of pathogens by the body and facilitating escape of HPV infection from the immune system. Hasimu et al. ${ }^{72}$ found that TLR4, 7 and 9 expression changes significantly in cervical cancer tissue. In addition, the expression levels of TLR4 and TLR9 were positively correlated with HPV16 infection. Altogether, these findings suggested that TLR4 and TLR9 may be the receptors for HPV infection. However, the exact roles of TLR4 and TLR9 in immune escape and the key molecular pathways involved remain unclear, and further study will be needed.

HPV can escape from TLR to invade the innate immune system. The HPV16 E6 protein can enhance the expression of functional components of the NF-KB signaling pathway, including p50, NIK and TRAF-interacting protein, and increase their binding to NF-KB and AP-1 DNA consensus binding sites. The studies of Lee et al. suggested that the expression levels of TLR9 gradually increased from cervical dysplasia to invasive cervical cancer tissues. However, the study did not further clarify the relationship between the increased TLR9 and cancer. ${ }^{73} \mathrm{Yu}$ et al. ${ }^{74}$ thought that the progression of cervical cancer was related to TLR4 due to frequent exposure to a variety of bacteria. However, they also observed that TLR4 expression declined during the progression of cervical cancer, and this downregulation of TLR4 appeared to be 
associated with the expression of P16INK4A, which is a crucial marker of HPV integration into host cells. ${ }^{74}$ These data may offer further insight regarding the association of HPV infection and TLR signaling during the development of cervical cancer.

However, our previous study found that TLR4 trended upward with increased disease severity after HPV infection, and the difference was statistically significant. ${ }^{40}$ The expression of HPV16 E7 in cervical lesions positively correlated with the severity of the lesion, but the difference was not significant. The correlation analysis showed that the expression of TLR4 in cervical cancer is irrelevant to HPV16 E7 $(r=0.121, P=0.0612)$. Our results also showed that the occurrence and development of cervical cancer were associated with the expression of TLR4 in HPV16-infected cervical lesions, but the severity of cervical lesions did not necessarily correlate with gene expression. This observation suggests that cytokine production regulated by other pathogens mediated by TLR4 may be involved indirectly in the process of persistent HPV infection, and that the immune response was synergistic with HPV infection through the TLR4 pathway to lead to tumor development. Since the high expression of TLR4 is closely linked with the development of cervical cancer but is not mediated by HPV, we reasoned that other pathogens, such as bacteria, may activate the TLR4 pathway to facilitate immune escape, which promotes the development of cervical lesions. However, the relationship between TLR4 and HPV infection, and whether the inhibition of TLR4 signaling pathway could inhibit cervical lesions after HPV infection, requires further study.

\section{HPV VACCINES AND TLR AGONISTS AS AN ADJUVANT IN TREATMENT OF CANCER}

HPV infection is currently the only clear cause of cervical cancer. The HPV prophylactic vaccine is a major breakthrough for cervical cancer prevention. However, it still faces some challenges in clinical practice. At present, improving the efficiency of the HPV vaccine is an area of active investigation. The HPV therapeutic vaccine aims to reduce or eliminate tumor cells infected with HPV through cell immunity and humoral immunity and to prevent precancerous lesions from continuing to deteriorate. However, a therapeutic vaccine was disappointing, demonstrating severe adverse reactions and having difficulty in recognizing tumorspecific target antigens. ${ }^{75}$ The persistent expression of HPV16 E6 and E7 protein is essential for tumor cell transformation and maintenance of malignancy in most cervical cancers. Moreover, precancerous lesions associated with HPV16 E6 and E7 protein can stimulate anti-tumor Cytotoxic $\mathrm{T}$ lymphocyte (CTL)-mediated cytotoxicity. Therefore the E6 and E7 protein are seen as tumorspecific antigens and are the focus of research and development of an HPV therapeutic vaccine. Many groups, including us, developed HPV16 E6 monoclonal antibodies, which provide a convenient tool for the quick detection of E6 protein. ${ }^{76}$ Furthermore, E6 protein structure-function analysis can clarify its role in cervical cancer development and facilitate preparation of a therapeutic HPV vaccine.

Gardasil and Cervarix are considered prophylactic cancer vaccines, as they have been developed to prevent infection with HPV serotypes that are associated with almost $70 \%$ of cervical cancer cases. ${ }^{75}$ Rosenberg et al. ${ }^{77}$ reported that the objective response rate was low (2.6\%) in a clinical trial with 440 cancer patients, with a lack of powerful adjuvants capable of overcoming the immunosuppression present in cancer patients. Thus, adjuvants are required to induce potent and durable immune response.

Molecules that can activate TLRs may be utilized for vaccine development and act as vaccine adjuvants. At present, the research and development of novel vaccine adjuvants are mainly focused on TLR ligands. Targeting TLR signaling pathways has been applied in clinical practice to improve the immunogenicity of
DNA vaccines and promote the adjustment of $T$ cells in resisting viral infection or to inhibit the wide spread inflammatory response caused by bacterial infection. ${ }^{78}$ Investigations showed that simultaneous activation of multiple pathways of TLRs by vaccines resulted in better immunogenicity effects. The high immunogenicity of the yellow fever vaccine is related to activation of multiple TLR signaling pathways in DCs. Activation of TLR signaling pathways can not only induce inflammatory reactions, but also can promote DC maturation and Th cell differentiation and produce an acquired immune response. Activation of TLR signaling pathways can be found not only in the immune response mechanism of the HPV vaccine, but also immune response mechanism of the rubella, measles, and hepatitis $B$ vaccines. $^{79}$ Vaccines and natural pathogens, in activating the pathogenic molecular activation pattern recognition receptor (PRR) pathway, induce innate immunity and are captured by DCs or other antigen-presenting cells in immune organs. The antigens are presented to $\mathrm{B}$ and $\mathrm{T}$ lymphocytes and produce an acquired immune response.

The distribution of different subtypes of TLRs differs among cells. Many studies found an important role of TLR pathways in infectious diseases and identified therapeutic advantages of novel agonists and antagonist ligands targeting TLRs. Hedayat et al. investigated the potential application of TLR ligands as vaccines or drugs. Modulators of TLR signal processing have been applied in clinical practice to improve vaccine effectiveness and promote Th1-type immune responses in viral or bacterial infection. ${ }^{77}$

Presently, only three TLR agonists are approved by international regulatory agencies for use in cancer patients: monophosphoryl lipid A (MPL), bacillus Calmette-Guérin (BCG), and imiquimod. ${ }^{81,82}$ MPL is a TLR4 ligand. Similar to LPS, it can activate the TRAM and TRIF signaling pathways and also can reduce the MYD-88 dependent signal pathway significantly that promoting inflammation. ${ }^{80}$

TLR binding of ligand can promote the differentiation and maturation of dendritic cells. It can stimulate the expression of molecules on the surface of the antigen-presenting cells and promote the production of cytokines. TLR signaling can also stimulate the transformation of naïve $T$ cells into regulatory $T$ cells and enhance the long-term protection of the host immune system. ${ }^{83}$ Pre-clinical trials in mice showed that glucopyranosyl lipid A (GLA) can function as a strong Th1 adjuvant that can also stimulate potent cellular immune responses. ${ }^{84}$ However, the only TLR4 agonist approved for human application is monophosphoryl lipid A (MPL), which has been tested in many clinical trials as a cancer vaccine adjuvant. ${ }^{85}$

Adjuvant System 04 (AS04) combines MPL and aluminum salt. A study finds that ASO4-induced innate responses are primarily due to $M P L$, and aluminum can extend the cytokine responses to MPL. ASO4 enhances vaccine response by rapidly triggering a local cytokine response, leading to an optimal activation of antigen-presenting cells. ${ }^{86} \mathrm{~A}$ clinical trial of the Cervarix vaccine with aluminum-MPL adjuvant and of the Gardasil vaccine with aluminum shows that MPL can enhance the effectiveness of the vaccine. The trial also shows that induced neutralization antibody titers were higher with Cervarix plus aluminum-MPL than with Gardasil plus aluminum. ${ }^{87}$ Compared with Gardasil, memory B cells in the blood increased significantly after HPV16/18 serum negative women receiving three immunizations with Cervarix. ${ }^{88}$

Currently, some clinical trials have indicated that vaccines administered with MPL as an adjuvant are safe and immunogenic. ${ }^{89}$ Moreover, the TLR7 agonist, TMX-101 (Vesimune), is currently being tested as an immunotherapeutic agent in a phase II clinical trial for noninvasive bladder cancer patients. ${ }^{90}$ Furthermore, several human clinical trials evaluating the adjuvant activities of CpG ODNs demonstrated that CpG ODNs can induce Th1-type immune responses, thereby becoming potential cancer 
vaccine adjuvants. Clinical trials using $C p G$ ODNs as immunotherapeutic agents in cancer patients suggested that CpG ODN as monotherapy or in combination with chemotherapy can induce potent anti-tumor immune responses that correlate with clinical benefit. $^{91,92}$ However, the potency of CpG ODN as a cancer vaccine adjuvant or anti-tumor agent needs further investigation.

Recently, adjuvant systems using different combinations of TLR adjuvants, including alum, MPL, and CPG ODN, have shown better efficacy compared with a single TLR adjuvant, ${ }^{93}$ suggesting that identifying optimal combinations of current adjuvants may be a better means of developing novel adjuvants for cervical cancer immunotherapy.

\section{CONCLUSION}

The control of cervical cancer includes the prevention of primary HPV infection by vaccination and the detection and treatment of cervical precancerous lesions. HPV infection and immune escape lead to persistent HPV infection and eventually to cervical cancer. However, the immune escape mechanism of HPV infection is very complex, involving a variety of cellular and molecular interactions, and needs further investigation. TLRs play extremely important roles in innate and acquired immunity and are also key regulatory factors in tumor development.

At present, many signaling pathways are under intensive investigation, but there is no clear mechanism. Although studies have shown that TLRs have changed cervical cancer infected with HPV, the significance of this change and whether HPV infection can provide a new target for the treatment of cervical cancer still requires further study. Some studies also showed that there may be other pathogens that promote cervical lesions after HPV infection, and these may be coordinated with the TLR4 signaling pathway. TLR4-induced HIF-1a may be involved in tumor immune escape as well, providing a new concept for the further analysis of HPV pathogenesis. The balance between virus and immune factors provides key information for HPV-associated cervical cancer prevention and treatment with vaccines. Our team prepared an E6 protein monoclonal antibody for the preparation of therapeutic HPV vaccines. TLRs as molecular adjuvants provide a new target for HPV infection prevention and provide direction for development of efficient vaccines. Since TLRs are of great significance in the response to HPV vaccines, further studies on the TLR family are warranted.

\section{ACKNOWLEDGEMENTS}

The authors thank Accixx Biomedical Consulting (www.Accixx.com) for editorial assistance. This work is supported by Sandy Rollman Ovarian Cancer Foundation, University of Pennsylvania BCPG Program, University of Pennsylvania URF pilot grant, National Natural Science Foundation of China (81302273), Health and family Planning Commission of Hubei Province, China (WJ2015MB084), Health Department of Hubei Province, China (2012ZY02) and Science and Technology Department Support Project of Hubei Province, China (2015BCA313).

\section{COMPETING INTERESTS}

The authors declare no conflict of interest.

\section{REFERENCES}

1 Peng Y, Fu ZZ, Guo CS, Zhang YX, Di Y, Jiang B et al. Effects and Mechanism of Baicalin on Apoptosis of Cervical Cancer HeLa Cells I $\mathrm{n}-\mathrm{v}$ itro. Iran J Pharm Res 2015; 14: 251-261.

2 Schiffman M, Castle PE, Jeronimo J, Rodriguez AC, Wacholder S. Human papillomavirus and cervical cancer. Lancet 2007; 370: 890-907.

3 Kawai T, Akira S. Toll-like receptors and their crosstalk with other innate receptors in infection and immunity. Immunity 2011; 34: 637-650.
4 Godfroy Jl 3rd, Roostan M, Moroz YS, Korendovych IV, Yin H. Isolated Toll-like receptor transmembrane domains are capable of oligomerization. PLoS One 2012; 7: e48875.

5 Iwasaki A, Medzhitov R. Regulation of adaptive immunity by the innate immune system. Science 2010; 327: 291-295.

6 Takeda K, Akira S. Toll-like receptors in innate immunity. Int Immunol 2005; 17: $1-14$.

7 Ravishankar Ram M, Goh KL, Leow AH, Poh BH, Loke MF, Harrison R et al. Polymorphisms at Locus 4p14 of Toll-Like Receptors TLR-1 and TLR-10 Confer Susceptibility to Gastric Carcinoma in Helicobacter pylori Infection. PLoS One 2015; 10: e0141865.

8 Al-Harras MF, Houssen ME, Shaker ME, Farag K, Farouk O, Monir R et al. Polymorphisms of glutathione S-transferase pi 1 and toll-like receptors 2 and 9: Association with breast cancer susceptibility. Oncol Lett 2016; 11: 2182-2188.

9 Luo Y, Jiang QW, Wu JY, Qiu JG, Zhang WJ, Mei XL et al. Regulation of migration and invasion by Toll-like receptor-9 signaling network in prostate cancer. Oncotarget 2015; 6: 22564-22574.

10 El-Omar EM, Ng MT, Hold GL. Polymorphisms in Toll-like receptor genes and risk of cancer. Oncogene 2008; 27: 244-252.

11 Ibrahim ZA, Armour CL, Phipps S, Sukkar MB. RAGE and TLRs: relatives, friends or neighbours? Mol Immunol 2013; 56: 739-744.

12 Tanaka T, Legat A, Adam E, Steuve J, Gatot JS, Vandenbranden M et al. DiC14amidine cationic liposomes stimulate myeloid dendritic cells through Toll-like receptor 4. Eur J Immunol 2008; 38: 1351-1357.

13 Alexopoulou L, Holt AC, Medzhitov R, Flavell RA. Recognition of double-stranded RNA and activation of NF-kappaB by Toll-like receptor 3. Nature 2001; 413: 732-738.

14 Kawai T, Adachi O, Ogawa T, Takeda K, Akira S. Unresponsiveness of MyD88deficient mice to endotoxin. Immunity 1999; 11: 115-122.

15 Kawai T, Takeuchi O, Fujita T, Inoue J, Muhlradt PF, Sato S et al. Lipopolysaccharide stimulates the MyD88-independent pathway and results in activation of IFNregulatory factor 3 and the expression of a subset of lipopolysaccharideinducible genes. J Immunol 2001; 167: 5887-5894.

16 Hoshino K, Kaisho T, Iwabe T, Takeuchi O, Akira S. Differential involvement of IFNbeta in Toll-like receptor-stimulated dendritic cell activation. Int Immunol 2002; 14: 1225-1231.

17 Rakoff-Nahoum S, Medzhitov R. Toll-like receptors and cancer. Nat Rev Cancer 2009; 9: 57-63.

18 Smith RL, Hebert HL, Massey J, Bowes J, Marzo-Ortega H, Ho P et al. Association of Toll-like receptor 4 (TLR4) with chronic plaque type psoriasis and psoriatic arthritis. Arch Dermatol Res 2016; 308: 201-205.

19 Catrysse L, Vereecke L, Beyaert R, van Loo G. A20 in inflammation and autoimmunity. Trends Immunol 2014; 35: 22-31.

20 Rawlings DJ, Dai X, Buckner JH. The role of PTPN22 risk variant in the development of autoimmunity: finding common ground between mouse and human. J Immunol 2015; 194: 2977-2984.

21 Cantley LC. The phosphoinositide 3-kinase pathway. Science 2002; 296: 1655-1657.

22 Hamerman JA, Pottle J, Ni M, He Y, Zhang ZY, Buckner JH. Negative regulation of TLR signaling in myeloid cells--implications for autoimmune diseases. Immunol Rev 2016; 269: 212-227.

23 Fukata M, Abreu MT. Role of Toll-like receptors in gastrointestinal malignancies. Oncogene 2008; 27: 234-243.

24 Pimentel-Nunes P, Afonso L, Lopes P, Roncon-Albuquerque Jr R, Goncalves N, Henrique $R$ et al. Increased expression of toll-like receptors (TLR) 2, 4 and 5 in gastric dysplasia. Pathol Oncol Res 2011; 17: 677-683.

25 Tsimogiannis KE, Tellis CC, Tselepis AD, Pappas-Gogos GK, Tsimoyiannis EC, Basdanis $G$. Toll-like receptors in the inflammatory response during open and laparoscopic colectomy for colorectal cancer. Surg Endosc 2012; 26: 330-336.

26 Eiro N, Gonzalez L, Gonzalez LO, Andicoechea A, Fernandez-Diaz M, Altadill A et al. Study of the expression of toll-like receptors in different histological types of colorectal polyps and their relationship with colorectal cancer. J Clin Immunol 2012; 32: 848-854.

27 Woods DC, White YA, Dau C, Johnson AL. TLR4 activates NF-kappaB in human ovarian granulosa tumor cells. Biochem Biophys Res Commun 2011; 409: 675-680.

28 Samara KD, Antoniou KM, Karagiannis K, Margaritopoulos G, Lasithiotaki I, Koutala $\mathrm{E}$ et al. Expression profiles of Toll-like receptors in non-small cell lung cancer and idiopathic pulmonary fibrosis. Int J Oncol 2012; 40: 1397-1404.

29 Gonzalez-Reyes S, Fernandez JM, Gonzalez LO, Aguirre A, Suarez A, Gonzalez JM et al. Study of TLR3, TLR4, and TLR9 in prostate carcinomas and their association with biochemical recurrence. Cancer Immunol Immunother 2011; 60: 217-226.

30 Theodoropoulos GE, Saridakis V, Karantanos T, Michalopoulos NV, Zagouri F, Kontogianni $\mathrm{P}$ et al. Toll-like receptors gene polymorphisms may confer increased susceptibility to breast cancer development. Breast 2012; 21: 534-538. 
31 Cai Z, Sanchez A, Shi Z, Zhang T, Liu M, Zhang D. Activation of Toll-like receptor 5 on breast cancer cells by flagellin suppresses cell proliferation and tumor growth. Cancer Res 2011; 71: 2466-2475.

32 Roh YS, Seki E. Toll-like receptors in alcoholic liver disease, non-alcoholic steatohepatitis and carcinogenesis. J Gastroenterol Hepatol 2013; 28(Suppl 1): 38-42.

33 Junjie X, Songyao J, Minmin S, Yanyan S, Baiyong S, Xiaxing D et al. The association between Toll-like receptor 2 single-nucleotide polymorphisms and hepatocellular carcinoma susceptibility. BMC Cancer 2012; 12: 57.

34 Grimmig T, Moench R, Kreckel J, Haack S, Rueckert F, Rehder R et al. Toll like receptor 2, 4, and 9 signaling promotes autoregulative tumor cell growth and VEGF/PDGF expression in human pancreatic cancer. Int J Mol Sci 2016; 17: 1-19.

35 de Matos LG, Candido EB, Vidigal PV, Bordoni PH, Lamaita RM, Carneiro MM et al. Association between Toll-like receptor and tumor necrosis factor immunological pathways in uterine cervical neoplasms. Tumori 2016; 103: 81-86.

36 Kelly MG, Alvero AB, Chen R, Silasi DA, Abrahams VM, Chan S et al. TLR-4 signaling promotes tumor growth and paclitaxel chemoresistance in ovarian cancer. Cancer Res 2006; 66: 3859-3868.

37 Ehrchen JM, Sunderkotter C, Foell D, Vogl T, Roth J. The endogenous Toll-like receptor 4 agonist S100A8/S100A9 (calprotectin) as innate amplifier of infection, autoimmunity, and cancer. J Leukoc Biol 2009; 86: 557-566.

38 Hasan UA, Bates E, Takeshita F, Biliato A, Accardi R, Bouvard V et al. TLR9 expression and function is abolished by the cervical cancer-associated human papillomavirus type 16. J Immunol 2007; 178: 3186-3197.

39 DeCarlo CA, Rosa B, Jackson R, Niccoli S, Escott NG, Zehbe I. Toll-like receptor transcriptome in the HPV-positive cervical cancer microenvironment. Clin Dev Immunol 2012; 2012: 785825.

40 Yanxiang Cheng GC, Wang X, Huang Y, Ding J, Huang J, Hong L. TLR4 may accelerate hypoxia reaction to promote the occurrence and progress of cervical lesions by infected pathogenic microorganisms other than HPV. J Cancer Ther 2013; 4: 549-553.

41 Lu Y, Hu J, Sun W, Duan X, Chen X. Hypoxia-mediated immune evasion of pancreatic carcinoma cells. Mol Med Rep 2015; 11: 3666-3672.

42 Tannahill GM, Curtis AM, Adamik J, Palsson-McDermott EM, McGettrick AF, Goel G et al. Succinate is an inflammatory signal that induces IL-1beta through HIF-1alpha. Nature 2013; 496: 238-242.

43 Kang GJ, Han SC, Yoon WJ, Koh YS, Hyun JW, Kang HK et al. Sargaquinoic acid isolated from Sargassum siliquastrum inhibits lipopolysaccharide-induced nitric oxide production in macrophages via modulation of nuclear factor-kappaB and c-Jun N-terminal kinase pathways. Immunopharmacol Immunotoxicol 2013; 35: 80-87.

44 Rahkola P, Mikkola TS, Ylikorkala O, Vaisanen-Tommiska M. Association between high risk papillomavirus DNA and nitric oxide release in the human uterine cervix. Gynecol Oncol 2009; 114: 323-326.

45 Tavares-Murta BM, de Resende AD, Cunha FQ, Murta EF. Local profile of cytokines and nitric oxide in patients with bacterial vaginosis and cervical intraepithelial neoplasia. Eur J Obstet Gynecol Reprod Biol 2008; 138: 93-99.

46 Fahey JM, Girotti AW. Accelerated migration and invasion of prostate cancer cells after a photodynamic therapy-like challenge: Role of nitric oxide. Nitric Oxide 2015; 49: 47-55.

47 Ranganathan S, Krishnan A, Sivasithambaram ND. Significance of twist and iNOS expression in human breast carcinoma. Mol Cell Biochem 2016; 412: 41-47.

48 Dong J, Cheng M, Sun H. Function of inducible nitric oxide synthase in the regulation of cervical cancer cell proliferation and the expression of vascular endothelial growth factor. Mol Med Rep 2014; 9: 583-589.

49 Xiao J, Guo Q, Wang X, Xie F, Zhang H, Sui L. [Study on the expression and signification of TLR4/NO pathway in cervical tumorigenesis with high risk HPV infection]. Zhonghua Fu Chan Ke Za Zhi 2015; 50: 41-47.

50 Tarcic O, Pateras IS, Cooks T, Shema E, Kanterman J, Ashkenazi H et al. RNF20 links histone $\mathrm{H} 2 \mathrm{~B}$ ubiquitylation with inflammation and inflammation-associated cancer. Cell Rep 2016; 14: 1462-1476.

51 Duan D, Zhang S, Li X, Guo H, Chen M, Zhang $Y$ et al. Activation of the TLR/ MyD88/NF-kappaB signal pathway contributes to changes in IL-4 and IL-12 production in piglet lymphocytes infected with porcine circovirus type 2 in vitro. PLoS One 2014; 9: e97653.

52 Ma XF, Zhang J, Shuai HL, Guan BZ, Luo X, Yan RL. IKKbeta/NF-kappaB mediated the low doses of bisphenol $A$ induced migration of cervical cancer cells. Arch Biochem Biophys 2015; 573: 52-58.

53 Matijevic T, Pavelic J. Toll-like receptors: cost or benefit for cancer? Curr Pharm Des 2010; 16: 1081-1090.

54 Lee JW, Choi JJ, Seo ES, Kim MJ, Kim WY, Choi CH et al. Increased toll-like receptor 9 expression in cervical neoplasia. Mol Carcinog 2007; 46: 941-947.

55 Cannella F, Pierangeli A, Scagnolari C, Cacciotti G, Tranquilli G, Stentella P et al. TLR9 is expressed in human papillomavirus-positive cervical cells and is overexpressed in persistent infections. Immunobiology 2015; 220: 363-368.
56 Jiang DS, Wang YW, Jiang J, Li SM, Liang SZ, Fang HY. MicroRNA-26a involved in Toll-like receptor 9mediated lung cancer growth and migration. Int J Mol Med 2014; 34: 307-312.

57 Di JM, Zhou J, Zhou XL, Gao X, Shao CQ, Pang J et al. Cyclooxygenase-2 expression is associated with vascular endothelial growth factor- $C$ and lymph node metastases in human prostate cancer. Arch Med Res 2009; 40: 268-275.

58 Bosch FX, Manos MM, Munoz N, Sherman M, Jansen AM, Peto J et al. Prevalence of human papillomavirus in cervical cancer: a worldwide perspective. International biological study on cervical cancer (IBSCC) Study Group. J Natl Cancer Inst 1995; 87: 796-802.

59 Walboomers JM, Jacobs MV, Manos MM, Bosch FX, Kummer JA, Shah KV et al. Human papillomavirus is a necessary cause of invasive cervical cancer worldwide. J Pathol 1999; 189: 12-19.

60 Garland SM, Brotherton JM, Condon JR, Mclntyre PB, Stevens MP, Smith DW et al. Human papillomavirus prevalence among indigenous and non-indigenous Australian women prior to a national HPV vaccination program. BMC Med 2011; 9: 104.

61 DelaRosa-Martinez R, Sanchez-Garza M, Lopez-Revilla R. HPV genotype distribution and anomalous association of HPV33 to cervical neoplastic lesions in San Luis Potosi, Mexico. Infect Agent Cancer 2016; 11: 16.

62 Yin FF, Wang N, Bi XN, Yu X, Xu XH, Wang YL et al. Serine/threonine kinases 31 (STK31) may be a novel cellular target gene for the HPV16 oncogene E7 with potential as a DNA hypomethylation biomarker in cervical cancer. Virol J 2016; 13: 60.

63 Yang X, Lu L. Expression of HPV-16 E6 protein and p53 inactivation increases the uterine cervical cancer invasion. Drug Res (Stuttg) 2015; 65: 70-73.

64 Garcia-Bates TM, Kim E, Concha-Benavente F, Trivedi S, Mailliard RB, Gambotto A et al. Enhanced cytotoxic CD8 $\mathrm{T}$ cell priming using dendritic cell-expressing human papillomavirus-16 E6/E7-p16INK4 fusion protein with sequenced antiprogrammed death-1. J Immunol 2016; 196: 2870-2878.

65 Woodworth CD. HPV innate immunity. Front Biosci 2002; 7: d2058-d2071.

66 Zanier K, ould M'hamed ould Sidi A, Boulade-Ladame C, Rybin V, Chappelle A, Atkinson A et al. Solution structure analysis of the HPV16 E6 oncoprotein reveals a self-association mechanism required for E6-mediated degradation of p53. Structure 2012; 20: 604-617.

67 Dionne KR, Zhuang Y, Leser JS, Tyler KL, Clarke P. Daxx upregulation within the cytoplasm of reovirus-infected cells is mediated by interferon and contributes to apoptosis. J Virol 2013; 87: 3447-3460.

68 White EA, Kramer RE, Tan MJ, Hayes SD, Harper JW, Howley PM. Comprehensive analysis of host cellular interactions with human papillomavirus E6 proteins identifies new E6 binding partners and reflects viral diversity. J Virol 2012; 86: 13174-13186.

69 Mantovani A, Allavena P, Sica A, Balkwill F. Cancer-related inflammation. Nature 2008; 454: 436-444.

70 Rahkola P, Vaisanen-Tommiska M, Tuomikoski P, Ylikorkala O, Mikkola TS. Cervical nitric oxide release and persistence of high-risk human papillomavirus in women. Int J Cancer 2011; 128: 2933-2937.

71 Daud II, Scott ME, Ma Y, Shiboski S, Farhat S, Moscicki AB. Association between toll-like receptor expression and human papillomavirus type 16 persistence. Int J Cancer 2011; 128: 879-886.

72 Hasimu A, Ge L, Li QZ, Zhang RP, Guo X. Expressions of Toll-like receptors 3, 4, 7, and 9 in cervical lesions and their correlation with HPV16 infection in Uighur women. Chin J Cancer 2011; 30: 344-350.

73 Nees M, Geoghegan JM, Hyman T, Frank S, Miller L, Woodworth CD. Papillomavirus type 16 oncogenes downregulate expression of interferon-responsive genes and upregulate proliferation-associated and NF-kappaB-responsive genes in cervical keratinocytes. J Virol 2001; 75: 4283-4296.

$74 \mathrm{Yu} \mathrm{L,} \mathrm{Wang} \mathrm{L,} \mathrm{Li} \mathrm{M,} \mathrm{Zhong} \mathrm{J,} \mathrm{Wang} \mathrm{Z,} \mathrm{Chen} \mathrm{S.} \mathrm{Expression} \mathrm{of} \mathrm{toll-like} \mathrm{receptor} 4$ is down-regulated during progression of cervical neoplasia. Cancer Immunol Immunother 2010; 59: 1021-1028.

75 Paavonen J, Naud P, Salmeron J, Wheeler CM, Chow SN, Apter D et al. Efficacy of human papillomavirus (HPV)-16/18 AS04-adjuvanted vaccine against cervical infection and precancer caused by oncogenic HPV types (PATRICIA): final analysis of a double-blind, randomised study in young women. Lancet 2009; 374: 301-314.

76 Yang $\mathrm{X}, \mathrm{CY}$. Preparation and detection of monoclonal antibody of human papillomavirus type 16 E6 protein. J Pract Diagn Ther 2016; 30: 552-555.

77 Rosenberg SA, Yang JC, Restifo NP. Cancer immunotherapy: moving beyond current vaccines. Nat Med 2004; 10: 909-915.

78 Hedayat M, Netea MG, Rezaei N. Targeting of Toll-like receptors: a decade of progress in combating infectious diseases. Lancet Infect Dis 2011; 11: 702-712.

79 Dabbagh K, Lewis DB. Toll-like receptors and T-helper-1/T-helper-2 responses. Curr Opin Infect Dis 2003; 16: 199-204.

80 Mata-Haro V, Cekic C, Martin M, Chilton PM, Casella CR, Mitchell TC. The vaccine adjuvant monophosphoryl lipid A as a TRIF-biased agonist of TLR4. Science 2007; 316: 1628-1632. 
81 Hemmi H, Kaisho T, Takeuchi O, Sato S, Sanjo H, Hoshino K et al. Small anti-viral compounds activate immune cells via the TLR7 MyD88-dependent signaling pathway. Nat Immunol 2002; 3: 196-200.

82 Hoffman ES, Smith RE, Renaud RC Jr. From the analyst's couch: TLR-targeted therapeutics. Nat Rev Drug Discov 2005; 4: 879-880.

83 Into T, Shibata K. Apoptosis signal-regulating kinase 1-mediated sustained p38 mitogen-activated protein kinase activation regulates mycoplasmal lipoproteinand staphylococcal peptidoglycan-triggered Toll-like receptor 2 signalling pathways. Cell Microbiol 2005; 7: 1305-1317.

84 Tagliamonte M, Petrizzo A, Tornesello ML, Buonaguro FM, Buonaguro L. Antigenspecific vaccines for cancer treatment. Hum Vaccin Immunother 2014; 10: 3332-3346.

85 Cluff CW. Monophosphoryl lipid A (MPL) as an adjuvant for anti-cancer vaccines: clinical results. Adv Exp Med Biol 2010; 667: 111-123.

86 Didierlaurent AM, Morel S, Lockman L, Giannini SL, Bisteau M, Carlsen H et al. AS04, an aluminum salt- and TLR4 agonist-based adjuvant system, induces a transient localized innate immune response leading to enhanced adaptive immunity. J Immunol 2009; 183: 6186-6197.

87 Einstein MH, Baron M, Levin MJ, Chatterjee A, Edwards RP, Zepp F et al. Comparison of the immunogenicity and safety of Cervarix and Gardasil human papillomavirus (HPV) cervical cancer vaccines in healthy women aged 1845 years. Hum Vaccin 2009; 5: 705-719.

88 Giannini SL, Hanon E, Moris P, Van Mechelen M, Morel S, Dessy F et al. Enhanced humoral and memory B cellular immunity using HPV16/18 L1 VLP vaccine formulated with the MPL/aluminium salt combination (AS04) compared to aluminium salt only. Vaccine 2006; 24: 5937-5949.

89 Neidhart J, Allen KO, Barlow DL, Carpenter M, Shaw DR, Triozzi PL et al. Immunization of colorectal cancer patients with recombinant baculovirus-derived KSA (Ep-CAM) formulated with monophosphoryl lipid A in liposomal emulsion, with and without granulocyte-macrophage colony-stimulating factor. Vaccine 2004; 22: 773-780.

90 Junt T, Barchet W. Translating nucleic acid-sensing pathways into therapies. Nat Rev Immunol 2015; 15: 529-544.
91 Pashenkov M, Goess G, Wagner C, Hormann M, Jandl T, Moser A et al. Phase II trial of a toll-like receptor 9-activating oligonucleotide in patients with metastatic melanoma. J Clin Oncol 2006; 24: 5716-5724.

92 Manegold C, Gravenor D, Woytowitz D, Mezger J, Hirsh V, Albert G et al. Randomized phase II trial of a toll-like receptor 9 agonist oligodeoxynucleotide, PF-3512676, in combination with first-line taxane plus platinum chemotherapy for advanced-stage non-small-cell lung cancer. J Clin Oncol 2008; 26: 3979-3986.

93 Garcon N, Chomez P, Van Mechelen M. GlaxoSmithKline adjuvant systems in vaccines: concepts, achievements and perspectives. Expert Rev Vaccines 2007; 6: 723-739.

94 O'Neill LA, Golenbock D, Bowie AG. The history of Toll-like receptors-redefining innate immunity. Nat Rev Immunol 2013; 13: 453-460.

95 Mohammad Hosseini A, Majidi J, Baradaran B, Yousefi M. Toll-Like Receptors in the Pathogenesis of Autoimmune Diseases. Adv Pharm Bull 2015; 5: 605-614.

96 Uematsu S, Jang MH, Chevrier N, Guo Z, Kumagai Y, Yamamoto M et al. Detection of pathogenic intestinal bacteria by Toll-like receptor 5 on intestinal CD11C+ lamina propria cells. Nat Immunol 2006; 7: 868-874.

97 Hemmi H, Takeuchi O, Kawai T, Kaisho T, Sato S, Sanjo H et al. A Toll-like receptor recognizes bacterial DNA. Nature 2000; 408: 740-745.

98 Hatai H, Lepelley A, Zeng W, Hayden MS, Ghosh S. Toll-like receptor 11 (TLR11) interacts with flagellin and profilin through disparate mechanisms. PLoS One 2016; 11: e0148987.

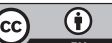

This work is licensed under a Creative Commons Attribution 4.0 International License. The images or other third party material in this article are included in the article's Creative Commons license, unless indicated otherwise in the credit line; if the material is not included under the Creative Commons license, users will need to obtain permission from the license holder to reproduce the material. To view a copy of this license, visit http://creativecommons.org/licenses/ by/4.0/

(c) The Author(s) 2017 